British Journal of Psychiatry (1992), 160, 563-574

\title{
Correspondence
}

Editor: Ian Pullen

Contents: Eating disorder and childhood sexual abuse/SLE and psychiatric morbidity/Head size in dementia/Adrenocortical suppression presenting with agitated depression, morbid jealousy, and a dementia-like state/Community care and the need for hospital beds/Family psychiatric histories in general practice referrals/Role of general practitioners in the care of long-term mentally ill patients/ Developing training themes from HRH's delivery/ Use of fluoxetine in heroin addiction/Violence on an acute ward/Continuation ECT/Familial delusional disorder linked with dyslexia/Capgras' syndrome in association with lithium toxicity.

\section{Eating disorder and childhood sexual abuse}

SIR: In his study of women with eating disorder, Waller (Journal, November 1991, 159, 664-671) compares the clinical features of those patients who reported sexual abuse with those who did not; the two groups are not compared in any other way. Yet there is no reason to assume that the rate of sexual abuse has been stable over time and is independent of factors such as parental social class; nor that the proportion of abused patients who are willing to report the experience to an interviewer is independent of, for example, age, education, social class, and marital status. If these factors are ignored there is a risk that spurious associations will be made between a history of abuse and various disorders.

Does a preconceived notion that sexual abuse is important in the genesis of mental disorder lead to its uncritical acceptance as a single aetiological variable in a way which would be unthinkable for a less emotive factor such as cerebral ventricular size? A search in the Journal and the American Journal of Psychiatry identified six studies in the past five years of essentially similar design to Dr Waller's, and also six studies which considered the computerised tomography (CT) scan finding of ventricular enlargement rather than the report of sexual abuse. Among the CT studies, five of the six authors reported that the ventricular size was independent of age; most also considered several other sociodemographic variables. By contrast, in the sexual abuse studies, only

two of the authors compared the subjects that reported abuse with those that did not in terms of any sociodemographic variables except sex. Yet such comparison would seem to be important: in both studies which did look for differences (Beck \& van der Kolk, 1987; Briere \& Zaidi, 1989), the groups reporting sexual abuse were significantly younger.

The body of literature concerning the long-term sequelae of sexual abuse is expanding: inevitably, much is based on the retrospective reports by patients. Untangling the complex links between abuse and psychopathology will be hampered if the report of sexual abuse is considered independently of other important variables.

BECK, J. C. \& VAN DER KOLK, B. (1987) Reports of childhood incest and current behaviour of chronically hospitalised psychotic women. American Journal of Psychiatry, 144, 1474-1476.

BRIERE, J. \& ZAIDI, L. (1989) Sexual abuse histories and sequelae in female psychatric emergency room patients. American Journal of Psychiatry, 146, 1602-1606.

John Conolly Hospital

JONATHAN M. GODFREY

Bristol Road

South Rednal

Birmingham B45 9BD

SIR: Waller (Journal, November 1991, 159, 664-671) has made a significant contribution to understanding the relationship between childhood sexual abuse and subsequent adult eating disorders. In particular, the interview protocol suggested should of be of general clinical use.

I would draw Dr Waller's attention to my small contribution to the literature (Journal, January 1988, 152, 145-146), which proposed a causal link between pre-morbid personality features, sexual assault in adolescence, and development of subsequent anorexia nervosa and other sexual adjustment difficulties.

Department of Community Psychiatry

D. M. HAMBIDGE

RAF Outpatients Department

Nocton Hall

Nocton

Lincoln LN4 2AA 